\title{
MiR-497 Suppresses YAP1 and Inhibits Tumor Growth in Non-Small Cell Lung Cancer
}

\author{
Chongbiao Huang ${ }^{\mathrm{a}}$ Ruijue Mab Jie Yue ${ }^{\mathrm{c}} \mathrm{Na} \mathrm{Li}^{\mathrm{d}}$ Zengxun Li ${ }^{\mathrm{d}}$ Daliang Qia \\ aSenior Ward, Tianjin Medical University Cancer Institute and Hospital, National Clinical Research \\ Center for Cancer, Key Laboratory of Cancer Prevention and Therapy, Tianjin, ${ }^{\circ}$ Tianjin Key Laboratory \\ of Ophthalmology and Visual Science, Tianjin Eye Hospital, Clinical College of Ophthalmology, \\ Tianjin Medical University, Tianjin, 'Department of Esophageal Carcinoma, Tianjin Medical University, \\ Cancer Institute and Hospital, National Clinical Research Center for Cancer, Key Laboratory of Cancer \\ Prevention and Therapy, Tianjin, dDepartment of Pancreatic Carcinoma, Tianjin Medical University, \\ Cancer Institute and Hospital, National Clinical Research Center for Cancer, Key Laboratory of Cancer \\ Prevention and Therapy, Tianjin, China
}

\section{Key Words}

MicroRNA • MiR-497 • Yes-associated protein $1 \cdot$ Non-small cell lung cancer • Proliferation

\begin{abstract}
Background/Aims: To investigate the expression, clinical significance and the cellular effects of miR-497 in non-small cell lung cancer (NSCLC). Methods: NSCLC cells were transiently transfected with miR-497 mimics or siRNA to up-regulate or down-regulate expression. Quantitative real-time PCR (qRT-PCR) was used to detect the mRNA level of miR-497. Luciferase assays, colony formation assays and BrdU incorporation assays were performed to identify the targets and role of miR-497 in NSCLC cells. Finally, the abundance of miR-497 was analyzed in a total of 51 NSCLC specimens. Results: The transcript levels of miR-497 were significantly decreased in NSCLC tissue (25/30; 83.3\%). Low miR-497 levels in tumor tissue correlated with advanced $\mathrm{pT}$ stage. Additionally, miR-497 transcript levels correlated with overall survival of NSCLC patients $(n=51, p=0.022)$. Overexpression of miR-497 inhibited the proliferation of NSCLC cell and down-regulation of miR-497 resulted in elevated NSCLC growth. Exogenous over-expression of YAP1 partially eliminated miR-497-induced cell growth. Conclusion: miR497 plays an important role in inhibiting the proliferation of NSCLC by targeting YAP1. Our results suggest that miR-497 is a potential therapeutic target in treating patients with NSCLC.
\end{abstract}

Copyright (C) 2015 S. Karger AG, Basel

\section{Introduction}

Lung cancer is one of the leading causes of cancer-related deaths worldwide [1]. NSCLC accounts for approximately 85\% of lung cancer [2]. Despite the different therapeutic C. Huang, R. Ma and J. Yue contributed equally to this work.

Daliang Qi

KARGER 125
Senior Ward, Tianjin Medical University, Cancer Institute and Hospital, Huanhu Xi Road, Hexi District, Tianjin, 300060, (China)

Tel. +86-022-23340123-2010, Fax+86-022-23340123-2010, E-Mail zlyygjbf@163.com 
strategies developed to date, the prognosis of NSCLC remains unsatisfactory. The 5-year survival rate of patients with NSCLC is less than $16 \%[3,4]$.

MicroRNAs (miRNAs) are a class of small non-protein-coding RNAs of approximately 22 nucleotides in size that are endogenously expressed in mammalian cells. miRNAs can posttranscriptionally regulate the expression of hundreds of target genes, thereby acting as oncogenes or tumor suppressors by modulating a wide range of biological functions such as cellular proliferation [5, 6], differentiation [7], metastasis [6, 8] and apoptosis [9]. MiR-497, a highly conserved miRNA located at Chromosome 17p13.1 [10], was recently found to play important inhibitory roles in malignancies by suppressing cancer cell proliferation [11] and inducing apoptosis [12].

Yes-associated protein 1 (YAP1), a key molecule of the Hippo signaling pathway, has been implicated as an oncogene in many types of malignancies [13]. YAP1 promotes proliferation and tumor growth by regulating several context-specific transcriptional programs [14]. Because YAP1 had been screened out as a potential target of miR-497, the possibility that miR-497 inhibits NSCLC proliferation via targeting YAP1 is intriguing.

In this study, we verified that miR-497 plays an inhibitory role in tumor growth in NSCLC cells as previously reported [15]. In addition, we explored the clinical significance of miR-497 in NSCLC patients and discovered that the expression level of miR-497 was associated with the overall survival of patients with NSCLC. The expression level of YAP1 in NSCLC tumor tissues was negatively associated with the level of miR-497. An interference of miR-497 promotes the proliferation of NSCLC cells, whereas ectopic expression of miR-497 results in inhibited proliferation activity by inhibiting YAP1.

\section{Materials and Methods}

\section{Cell lines and tumor specimens}

All of the NSCLC cell lines used in this study were bought from the Cell Culture Center of the Shanghai Institute for Biological Sciences (Chinese Academy of Science, Shanghai, China), including five adenocarcinoma cell lines (A549, H1299, H358, H1975 and H1395); two squamous cell lines (H520 and SKMES-1); and large cell carcinoma cell line, H460. The cancer cells were grown in monolayer in 1640 culture medium supplemented with $10 \%$ fetal bovine serum (FBS) and maintained at $37^{\circ} \mathrm{C}$ in humidified air with $5 \% \mathrm{CO} 2$.

A total of 51 tumor tissue specimens and 30 corresponding adjacent normal lung tissues were obtained through the Tumor Tissue Bank of Tianjin Cancer Hospital from patients who underwent curative resection for NSCLC at the Tianjin Cancer Institute and Hospital (TJMUCH) between 2007 and 2008. The median follow-up time for overall survival (OS) was 48 months. This study was approved by the Ethics Committee of TJMUCH. All patients signed a written consent for the use of their specimens and disease information for future investigations according to the ethics committee.

SiRNA, miRNA, plasmid construction, transfection, and luciferase assays

SiRNAs against miR-497 and YAP1 were designed as described [16, 17]. A scrambled siRNA sequence ( $5^{\prime}$-UUCUCCGAACGUGUCACGUTT- $3^{\prime}$ ) was used as a control. The overexpression of miR-497 mimics was described previously [18]. Non-targeting control (NTC) was used as a control. All of the RNAs were synthesized from Genepharma. The coding domain sequence of human YAP1 mRNA was cloned into pcDNA 3.1 vector (Invitrogen). For transfection, the cells were plated at a density of $5 \times 10^{5}$ cells/well in 6 -well plates. When the cells reached $80 \%$ confluence, 100 pmol of siRNA or $4 \mu \mathrm{g}$ of DNA were transfected into cells using Lipofectamine 3000 (Invitrogen) for 48 hours according to the manufacturer's instructions.

The 3'-untranslated region (UTR) of human YAP1 mRNA was amplified by PCR using the following primers: YAP1-3' UTR-F: 5'-ATG CAT AGT TTC TGC CCA AAGGT-3'; YAP1-3' UTR-R: 5'-GTT GCC TTT CCC CGC CTCCC-3', and cloned into the Xhol/NotI sites of the pmirGLO vector (Promega). All of the constructs were verified by sequencing. For the luciferase assays, HEK293T cells were seeded in 6-well plates and transfected with $4 \mu \mathrm{g}$ of the pmirGLO-YAP1 3'-UTR reporter vector per well and 100 pmol of microRNA or 


\section{Cellular Physiology Cell Physiol Biochem 2015;37:342-352 \begin{tabular}{l|l} 
and Biochemistry Published onIIne: August 24, 2015 & $\begin{array}{l}\text { C) 2015 S. Karger AG, Basel } \\
\text { www.karger.com/cpb }\end{array}$ \\
\hline
\end{tabular} \\ Huang et al.: MiR-497/YAP1 Axis Inhibits NSCLC Growth}

control RNA using Lipofectamine 3000 (Invitrogen). Site-specific mutants of the 3'-UTR reporters for YAP1 were also tested.

\section{Protein extraction and Western blot analysis}

Whole-cell extracts were prepared by lysing cells with SDS lysis buffer supplemented with a protease inhibitor cocktail (Sigma). A total of $20 \mu \mathrm{g}$ of protein lysates were separated by SDS-PAGE and then the target proteins were detected by Western blot analysis with the following primary antibodies: rabbit anti-human Yap1 monoclonal antibody (Abcam), and mouse anti-human Ki-67 polyclonal antibody (Abmart). After further washes, the membranes were incubated with the goat anti-rabbit/mouse peroxidase-conjugated secondary antibodies (Abcam), and the blots were developed using ECL (Millipore).

\section{RNA extraction and Real-time PCR}

Total RNA of the cells and the tissues was extracted using Trizol (Invitrogen) according to the manufacturer's instructions. Then, a total of $3 \mu \mathrm{g}$ of mRNA was reverse transcribed to single-stranded cDNAs using a reverse-transcription PCR (RT-PCR) system (TaKaRa). qRT-PCR for miR-497, YAP1 and c-myc were performed using SYBR premix real-time PCR Reagent (TaKaRa). The primers for miR-497, U6 and c-myc were listed elsewhere $[19,20]$. The primer sequences for YAP1 and $\beta$-actin were as follows: YAP1 (5' -AGA ACA ATG ACG ACC AAT AGCTC- 3', 3' -GCT GCT CAT GCT TAG TCCAC- 5'), $\beta$-actin (5' -CCT GGG CAT GGA GTC CTGTG- 3', 3' -AGG GGC CGG ACT CGT CATAC- 5'). The 25- $\mu$ L PCR reaction mixture contained: $2 \mu \mathrm{L}$ of reverse transcription product, $1 \times$ PCR Master Mix and $0.2 \mu \mathrm{mol} / \mathrm{L}$ forward and reverse primers. U6 RNA was used to normalize the miR-497 RNA levels and $\beta$-actin was used to normalize the level of other mRNAs. The results are presented as fold change in cells or tissues.

\section{Immunohistochemical staining}

Immunohistochemical staining for YAP1 of NSCLC patient tissues was performed according to the manufacturer's instructions. In brief, paraffin-embedded sections of NSCLC tissue micro arrays were deparaffinized and then heated in a pressure pot for 3 minutes for antigen retrieval. Then, the sections were incubated with rabbit anti-human YAP1 monoclonal antibody at a 1:500 dilution (Abcam) overnight at $4^{\circ} \mathrm{C}$. The slides were then incubated with a goat anti-rabbit/mouse secondary antibody (Maxin) at $37^{\circ} \mathrm{C}$ for 30 min. A DAB Substrate Kit (Maxin) was used carry out the chromogenic reaction. The results were scored by two experienced pathologic examiners who were unaware of the clinicopathologic data. The intensity of the YAP1 staining was evaluated using the following criteria: 0 , negative; 1 , low; 2 , medium; 3 , high. The extent of the staining was scored as $0,0 \%$ stained; $1,1 \%$ to $25 \%$ stained; $2,26 \%$ to $50 \%$ stained; and $3,51 \%$ to $100 \%$ stained. Five random fields (20× in magnification) were evaluated under a light microscope. The final scores were calculated by multiplying the scores of intensity with that of extent. The staining results were divided into two grades by final scores: 0 to 2, low staining; 3 to 9, high staining.

The colony formation assay and the 5'-bromo-2'-deoxyuridine (BrdU) incorporation assay

A colony formation assay was carried out as described previously [21]. In brief, a six-well plate was seeded at a density of 800 cells per well. The plates were maintained at $37^{\circ} \mathrm{C}$ in a humidified incubator, and the culture medium was replaced every 3 days. After 2 weeks, the cells were stained with $0.5 \%$ Crystal Violet and the average colony number of five random fields ( $4 \times$ in magnification) was counted under a microscope.

A BrdU ELISA kit (Roche) was also used to measure the proliferation activity [22]. Briefly, the cells were cultured in a 96-well plate overnight. Subsequently, BrdU was added to the culture medium for 2 hours. Then, the anti-BrdU-POD binds to the BrdU incorporated into newly synthesized DNA. Finally, the reaction product was quantified by the absorbance value (A370nm-A490nm).

\section{Statistical analysis}

Statistical analyses were carried out using the IBM SPSS Statistics Program 19.0 (Armonk, New York, the United States). Each experiment was performed in triplicate and the values are presented as the mean \pm SD. Student's t test for unpaired data was used to compare the mean values. Kaplan-Meier curves and log-rank tests were calculated for the YAP1 expression level. Pearson correlations were run to measure the association between pairs of variables. All of the probability values had a statistical power level of $90 \%$ and a 2 -sided level of $5 \%$. $\mathrm{P}<0.05$ was considered significant.

\section{KARGER}




\section{Results}

miR-497 inhibits cell proliferation in NSCLC

The expression level of miR-497 was detected in 8 NSCLC cell lines using qRT-PCR. Three cell lines (A1395, and SK-MES-1 and H520) had high levels of miR-497, whereas other cell lines (H358, A549, H1975, H1299 and H460) presented relative lower expression levels (Fig. 1A). To explore the role of miR-497 in proliferation, H520 (high miR-497 expression) and H1299 (low miR-497 expression) cells were transiently transfected with anti-miR-497 siRNA and miR-497 mimics, respectively. Then, the transfected cells were subjected to proliferation assays. BrdU incorporation assays were performed, and growth curves were drawn. As shown in Fig. 1B, H520 cells transfected with anti-miR-497 showed significantly higher growth activity than the control cells (H520 with scrambled RNA). The differences emerged at $24 \mathrm{~h}$ and peaked at $48 \mathrm{~h}$ after transfection. In contrast, the H1299 cells with the miR-497 mimic had significantly reduced proliferation activities compared to H1299 cells transfected with NTC at $48 \mathrm{~h}$ and $72 \mathrm{~h}$. H520 cells transfected with anti-miR-497 siRNAs displayed an elevated Ki-67 level, whereas H1299 cells transfected with miR-497 mimics showed decreased Ki-67 expression (Fig. 1C). Additionally, in the colony formation assays, the transfection of the miR-497 mimic reduced colonies (Fig. 1D, P<0.05), whereas antimiR-497 siRNAs increased the number of colonies (Fig. 1D, $\mathrm{P}<0.05$ ). Taken together, these data revealed a critical role of miR-497 in the proliferation of NSCLC cells.

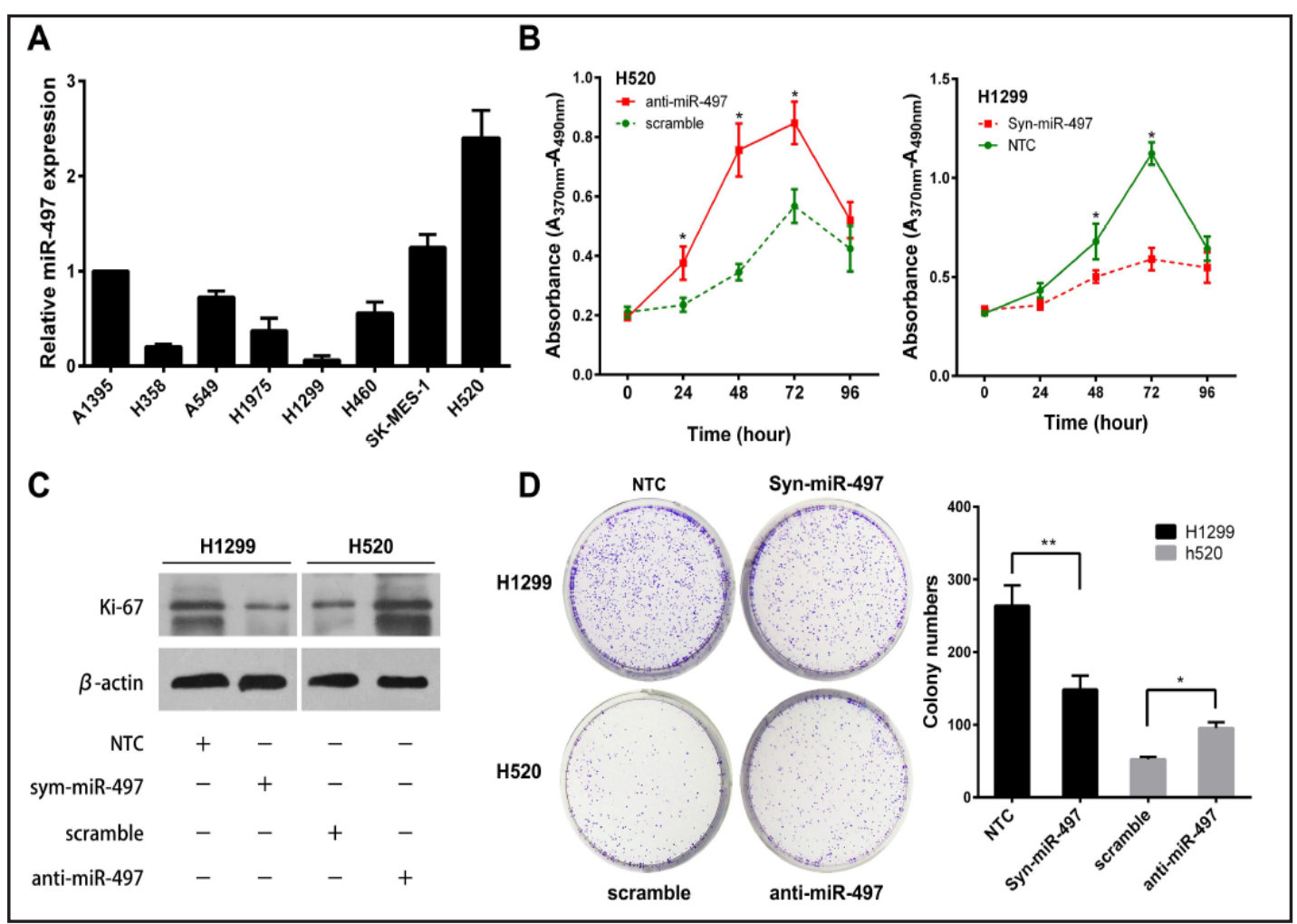

Fig. 1. miR-497 inhibits proliferation in NSCLC cell lines. A, miR-497 expression in 8 NSCLC cell lines was assayed using qRT-PCR. U6 was used as a control to normalize the CT values. The resulting value was then transformed by applying $2^{-\Delta C T}$. The mean \pm SD of normalized $C_{T}$ was from 3 independent experiments. $B$, proliferation activity was evaluated with BrdU incorporation assays in H520 and H1299 cells that were transiently transfected with siRNA and mimic for miR-497, respectively. Scrambled RNA and non-targeting control RNA was used as a control. The mean \pm SD of the absorbance was from 3 independent experiments. Forty-eight hours after transfection, H520 and H1299 cells were used to detect the proliferation marker, Ki67 (C), and the colony formation assay (D). The colonies were stained with crystal violet after an incubation for 2 weeks (left). The mean \pm SD of the colonies from 3 independent experiments is shown to the right. *, $P<0.05 ; * *, P<0.001$. 
Fig. 2. $\mathrm{miR}-497$ targets YAP1 through an interaction with the YAP1 3'-UTR. A, schematic diagram of the putative miR497 binding site in human YAP1 3'-UTR and luciferase constructs with wild-type and mutant (YAP 1-3'-UTR) miR-497 target sequences. The possible binding sites of miR-497 in the 3'-UTR of the target genes were searched in Targetscan (http: / / www. targetscan.org/). B, HEK293T cells were transfected with the luciferase constructs shown in $\mathrm{A}$ and 100 pmol of miR-497 mimics (syn-miR-497) or non-targeting control RNA (NTC). The transfection efficiency was validated using qRT-PCR (top). Forty-eight hours later, the cells were harvested for luciferase analysis (bottom). Renilla luciferase activity is normalized to firefly luciferase activity. C. HEK293T cells were transfected with the luciferase constructs shown in $\mathrm{A}$ and

A

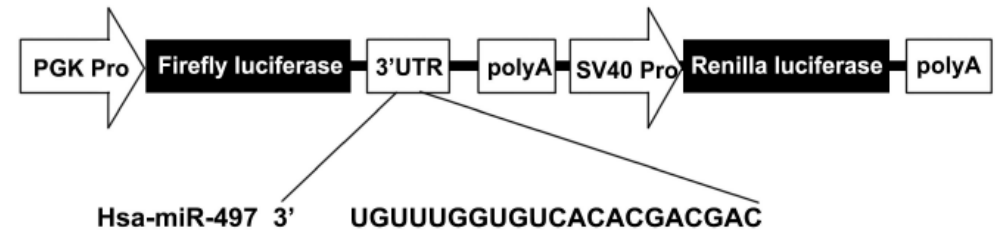

| | | | | |

Wt. YAP1 3' UTR 5' CUCUUCCUUGUCCAUUGCUGCUG Mut. YAP1 3' UTR 5' CUCUUCCUUGUCCAUGUACAUGU

B
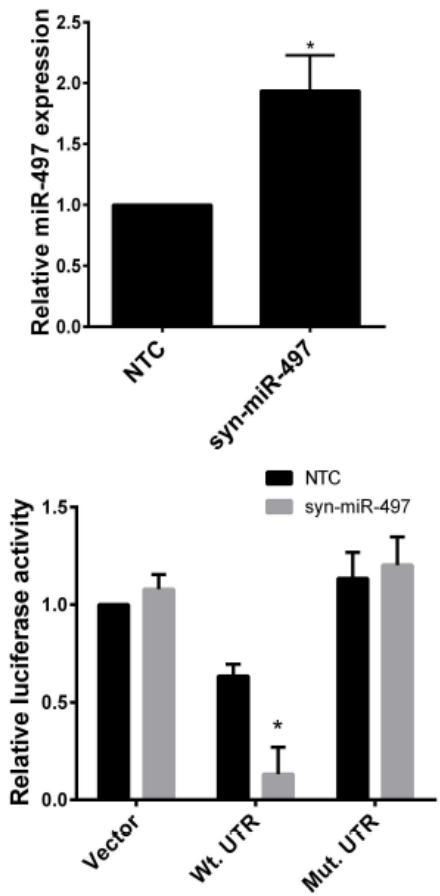

D

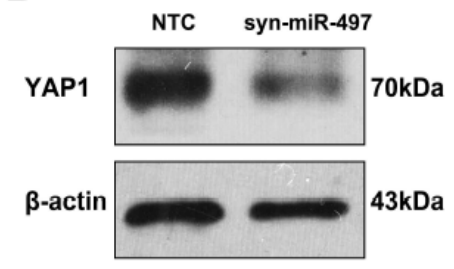

$F$

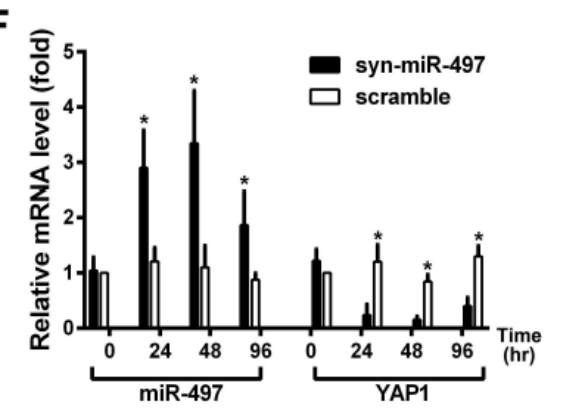

C

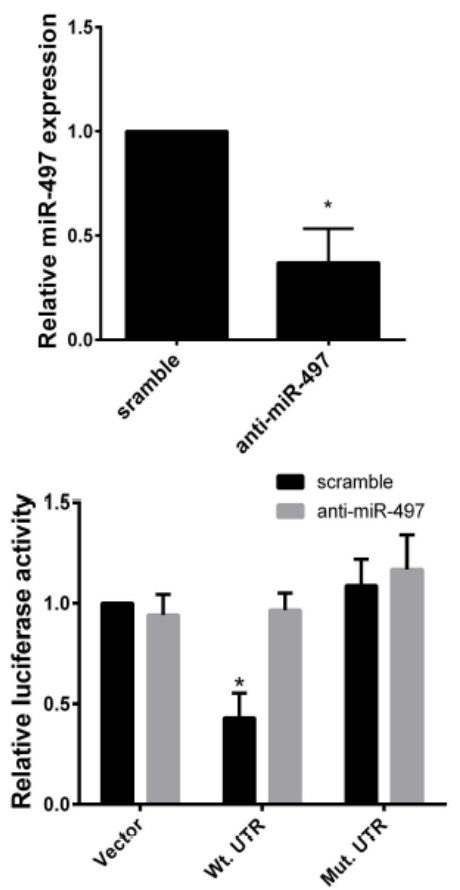

$\mathbf{E}$
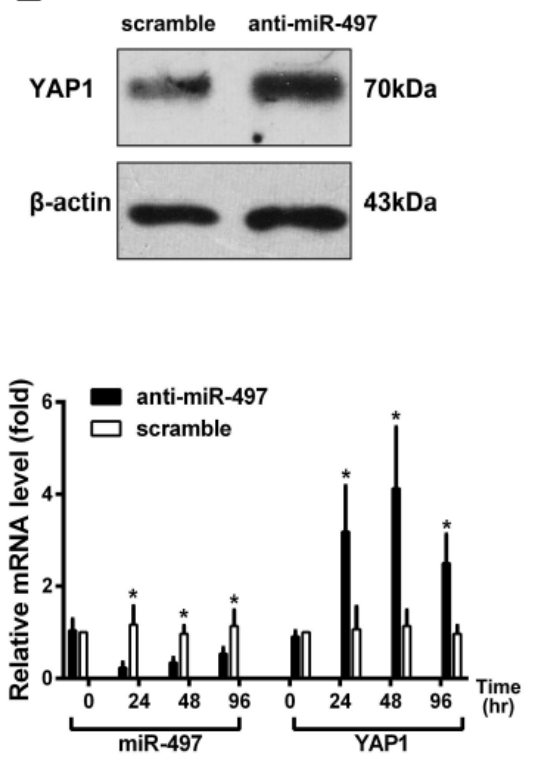
100 pmol of siRNA for miR-497 (anti-miR-497) or scrambled RNA. The transfection efficiency was validated using qRT-PCR (top). Forty-eight hours later the cells were harvested for luciferase analysis (bottom). Renilla luciferase activity is normalized to firefly luciferase activity. D. H1299 cells were transfected with synmiR-497 or non-targeting control RNA (NTC) and subjected to Western blotting after 48 hours. E. H520 cells were transfected with anti-miR-497 or scrambled RNA and subjected to Western blotting (bottom) after 48 hours. $\beta$-actin was used for a loading control in the Western blotting assays. F, H520 cells transiently transfected with miR-497 mimics (left) or siRNA against miR-497 (right). Scrambled RNA was used as a control. At the indicated time points, the cells were harvested and subjected to RT-PCR analysis for the mRNA level of miR-497 and YAP1. The relative mRNA levels were normalized to U6 and $\beta$-actin, respectively. The values are presented as the mean \pm SD of 3 independent experiments. ${ }^{*}, P<0.05$.

YAP1 is negatively regulated by miR-497

The 3483 nt-3'-UTR of YAP1 was screened out for complementarity with the sequence of miR-497 via a bioinformatic search in Targetscan. A 7mer-m8 (exact match to positions 2-8 of the mature miRNA) target sequence for miR-497 at nt 162-168 of the 3'-UTR of YAP1 was found.

A wild type or mutant 3'-UTR of the YAP1 gene was inserted into the dual luciferase vector pmirGLO (Fig. 2A). In addition to the luciferase plasmid, the miR-497 mimics or antimiR-497 SiRNAs were cotransfected into HEK293T cells. The transfection efficiency was validated using qRT-PCR (Fig. 2B \& 2C, top). The results of the luciferase assays showed that syn-miR-497 mimics significantly inhibited the luciferase activity of the wild type 3'UTR reporter gene by $79.1 \% \pm 6.7 \%$ in HEK293T cells. As expected, syn-miR-497 did not influence the luciferase activity of the mutant 3'-UTR reporter gene (Fig. 2B, bottom). In contrast, the luciferase activity of the reporter gene was significantly elevated when antimiR-497 siRNAs were cotransfected with the wild-type reporter (Fig. 2C, bottom). These results confirmed that YAP1 was a direct target gene of miR-497. In addition, transfection with the syn-miR-497 mimics led to a 58.3\% reduction of YAP1 protein expression in H1299 cells (Fig. 2D). In contrast, anti-miR-497 siRNAs elevated the protein level of YAP1 by $41.6 \%$ in H520 cells (Fig. 2E). Additionally, the mRNA level of YAP1 was negatively regulated by miR497 (Fig. 2F). Taken together, these data suggest that miR-497 suppresses YAP1 expression by directly targeting the 3'-UTR of the YAP1 gene.

miR-497 suppresses the proliferation of NSCLC by targeting YAP1

The correlation of the expression levels of miR-497 and YAP1 in 8 NSCLC cell lines was investigated. Five cell lines (H358, A549, H1975, H1299 and H460) had low miR-497 levels and high YAP1 levels. In contrast, the other three cell lines (A1395, SK-MES-1 and H520) had high miR-497 levels and low YAP1 levels. Pearson correlation analysis revealed that the level of YAP1 significantly correlated to that of miR-497 in these cell lines (Fig. 3A; rs $=-0.830, P$ $=0.011$ ).

Subsequently we investigated whether miR-497 inhibits proliferation by directly targeting YAP1. Syn-miR-497 and pcDNA 3.1-YAP1 were cotransfected into H1299 cells, with NTC and pcDNA 3.1 vector as controls. The proliferation activity of the transfected cells was assessed using BrdU assays (Fig. 3B). The results revealed that syn-miR-497 significantly reduced the proliferation activity of H1299 cells compared to NTC. However, pcDNA 3.1YAP1 could partially antagonize this growth-inhibiting effect of syn-miR-497. Moreover, the protein levels of c-myc, a downstream gene of YAP1 [23], as well as Ki-67, which is usually used as a proliferation marker [24], were detected 48 hours after cotransfection (Fig. 3C). As expected, the levels of c-myc and Ki-67 in H1299 cells cotransfected with pcDNA 3.1 and syn-miR-497 were lower than pcDNA 3.1 and NTC. Interestingly, when pcDNA 3.1-YAP1 was cotransfected with syn-miR-497, the levels of c-myc and Ki-67 were not reduced as much as in cells cotransfected with pcDNA 3.1 and syn-miR-497. These data suggested that miR-497 suppresses downstream gene expression and inhibits cell proliferation by targeting YAP1.

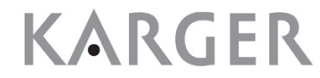




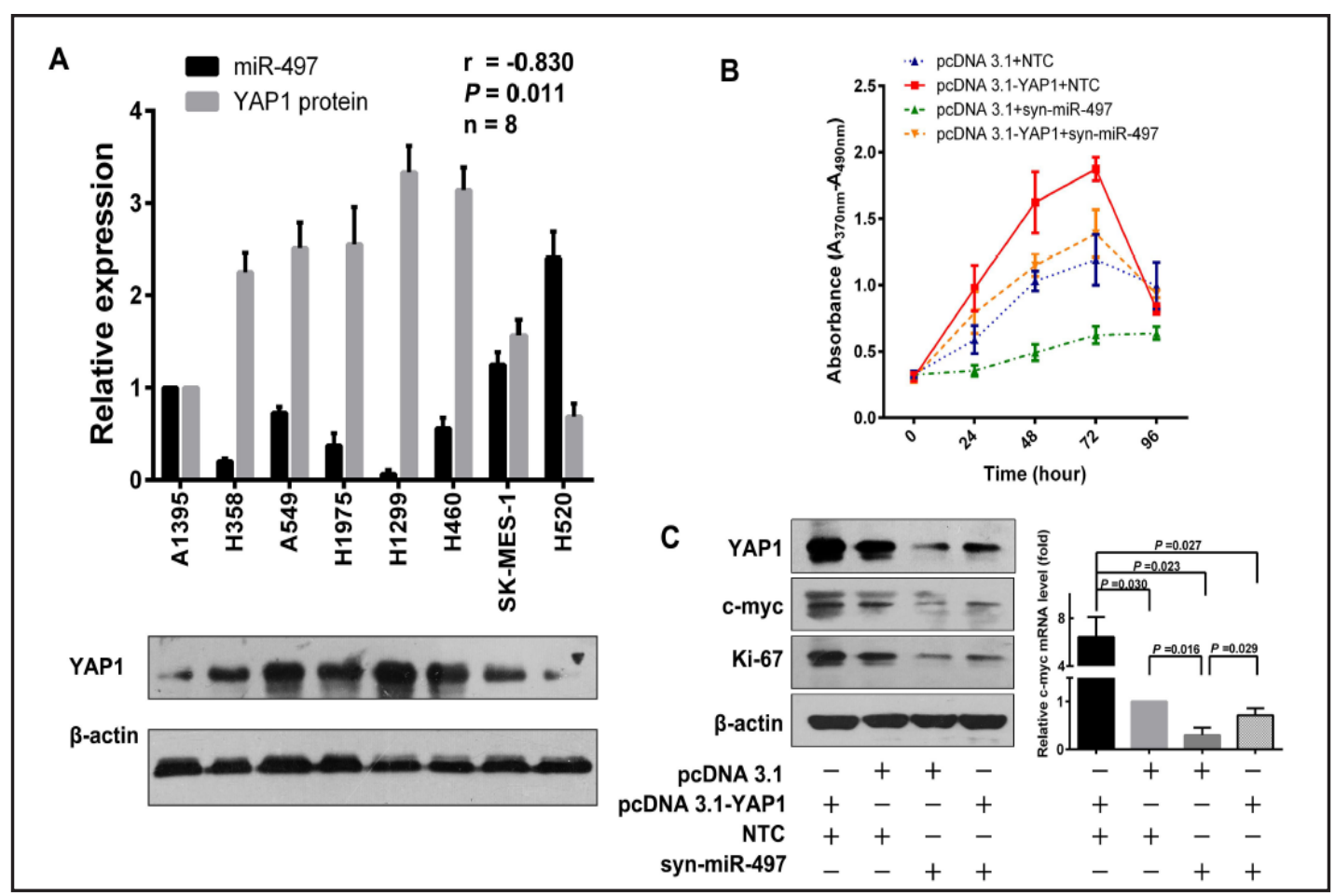

Fig. 3. miR-497 inhibits proliferation of NSCLC cells by targeting YAP1. A, relative expression levels of miR497 and YAP1 in 8 human NSCLC cell lines. Pearson correlation analysis was used to evaluate the correlation between YAP1 and miR-497 expression levels in these cell lines ( $\mathrm{n}=8 ; \mathrm{r}=-0.830 ; P=0.011)$. B, H1299 cells were cotransfected with pcDNA 3.1-YAP1/pcDNA 3.1 and syn-miR-497/NTC and then subjected to BrdU incorporation assays 48 hours later. C, H1299 cells were pretreated as in B but subjected to Western blotting assays (left) and real-time PCR analysis for the expression of c-myc (right). The protein levels of YAP1, c-myc and Ki-67 and the mRNA level of c-myc were normalized to $\beta$-actin. The data were from 3 replicated experiments.

\section{Inverse correlation between YAP1 and miR-497 expression in NSCLC patients}

To explore the expression of miR-497 in NSCLC tissues, we examined the miR-497 level in tumor tissues and the corresponding adjacent normal lung tissues in a total of 30 patients with NSCLC (Fig. 4A). The data showed that 25 of the 30 cases (83.3\%) had decreased miR497 levels in tumor tissues compared to the adjacent normal tissues. The expression levels of miR-497 and YAP1 were detected in tumor tissues of 51 patients with NSCLC by qRT-PCR and immunohistochemical staining, respectively (Fig. 4A). Pearson correlation analysis revealed that the expression of miR-497 was inversely correlated with YAP1 in the 51 patients (rs = -0.526; $P<0.001$; Fig.4C).

The relationship between the level of miR-497 and the clinicopathologic parameters of NSCLC are summarized in Table 1 . The 51 patients were stratified into two groups by the median value of the miR-497 level. No significant correlation was noticed between the miR497 level and age, gender, pathologic type, pN stating, or differentiation. However, pT staging had a significant association with the miR-497 level. In addition, we investigated the prognostic significance of miR-497 in these patients with NSCLC. Kaplan-Meier analysis revealed that the patients with a low miR-497 level had poorer survival (Fig. 4D). The median overall survival of the low miR-497 group and high miR-497 group was $25.08 \pm 3.67$ months and $31.64 \pm 2.08$ months, respectively $(P=0.022)$. These findings support our finding that miR-497 promotes NSCLC progression via targeting YAP1.

\section{KARGER}


A
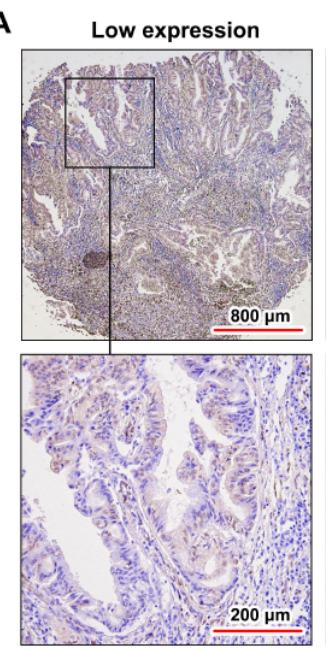

B

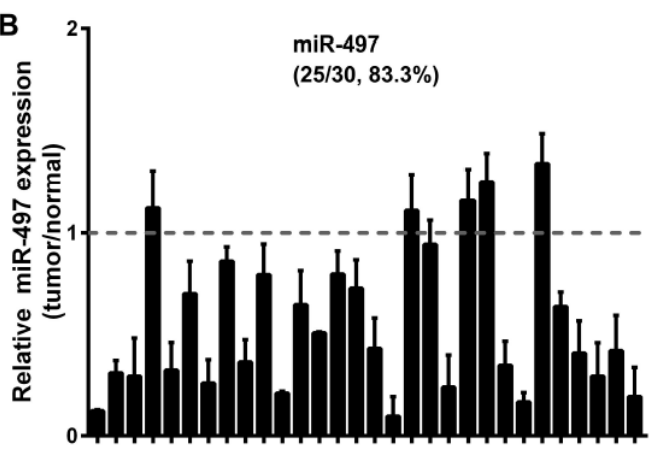

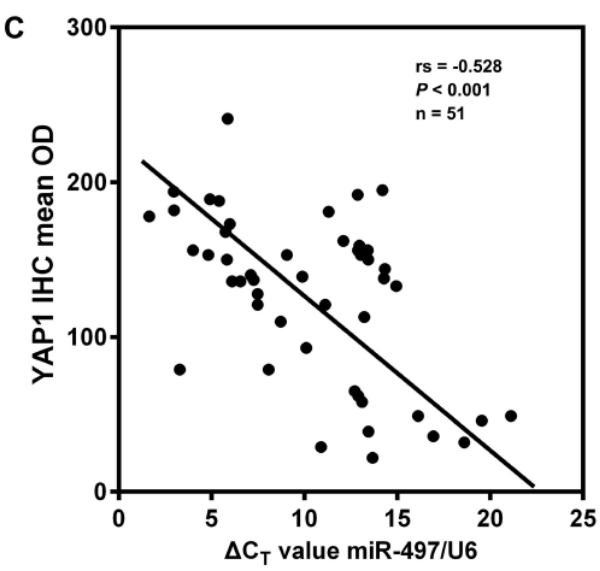

D

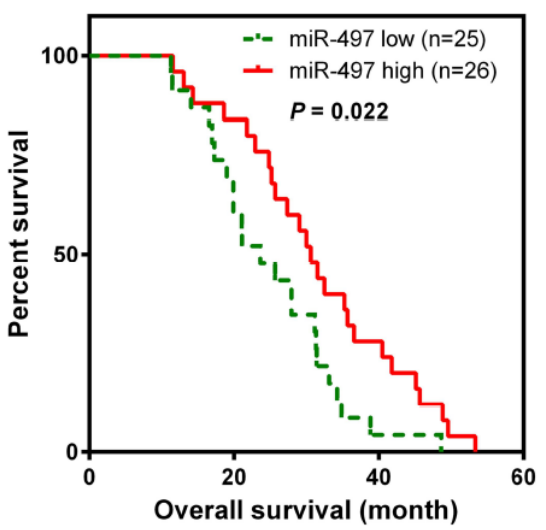

Fig. 4. Inverse correlation between YAP1 and miR-497 expression in NSCLC patients. A, immunohistochemical staining examples are shown for strong and low YAP1 expression in NSCLC tissues. Original magnification, $\times 200$. B, relative miR-497 expression in NSCLC specimens and the corresponding normal lung tissues from 30 patients with NSCLC. U6 was used to normalize the $\mathrm{C}_{\mathrm{T}}$ values. The results are presented as the division of the $\mathrm{C}_{\mathrm{T}}$ value of tumor specimens divided by the corresponding normal tissues. The mean $\pm \mathrm{SD}$ was from 3 independent experiments. C, Pearson analysis for the correlation of YAP1 and miR-497 expression levels in patients with NSCLC $(n=51 ; R=-0.528 ; p<0.001)$. YAP1 staining intensity from 24 random regions in A was quantified using ImageJ software. miR-497 expression levels were normalized to U6 and presented as the $\Delta \mathrm{C}_{\mathrm{T}}$ value. The mean $\pm \mathrm{SD}$ was calculated from 3 independent experiments. D. Kaplan-Meier analysis of survival of 51 patients with NSCLC ( $\mathrm{P}=0.022$ by log-rank test) according to the expression of miR-497. The vertical bars on the survival curves indicate censored cases.

\section{Discussion}

Here we report that miR-497 suppresses YAP1 expression by directly binding to the 3'UTR of the YAP1 gene. This study is the first to demonstrate the suppressive roles of miR-497 in the growth of NSCLC.

As a transcriptional coactivator and key mediator of the Hippo signaling pathway, YAP1 is posttranscriptionally regulated by either kinase-mediated degradation or cytoplasmic sequestration [23]. In addition to regulating organ development [24] and inducing epithelialmesenchymal transition [25] and tumorigenesis [20], YAP1 has been identified as a critical modulator of cancer proliferation. In their study Bin Zhao et al. showed that cellular contact induces the phosphorylation and cytoplasmic retention of Yap1 and leads to an evasion of contact inhibition of proliferation [26]. Li M et al. explored the clinical significance and biological functions of YAP1 in human gallbladder tumors and found that nuclear YAP1 and its target gene AXL were overexpressed in these tumor tissues [27]. 


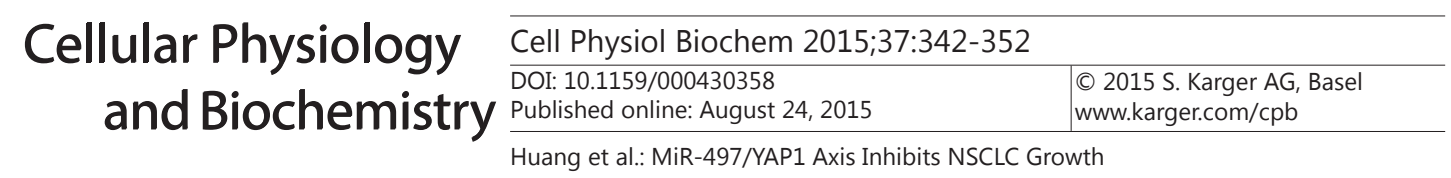

Table 1. Association between miR-497 expression and patients'clinicopathological parameters ( $\mathrm{n}=51)$. ADC, adenocarcinoma; SCC, squamous cell lung cancer

\begin{tabular}{|c|c|c|c|c|c|}
\hline & $\begin{array}{c}\text { Total } \\
(\mathrm{n}=51)\end{array}$ & $\begin{array}{c}\text { Low expression } \\
(\mathrm{n}=26)\end{array}$ & $\begin{array}{l}\text { High expression } \\
(\mathrm{n}=25)\end{array}$ & $x^{2}$ & $P$ value \\
\hline \multicolumn{6}{|l|}{ Gender } \\
\hline Male & 36 & 16 & 20 & 1.025 & 0.311 \\
\hline Female & 15 & 9 & 6 & & \\
\hline \multicolumn{6}{|l|}{ Age,y } \\
\hline$<65$ & 27 & 12 & 15 & 0.481 & 0.488 \\
\hline$\geq 65$ & 24 & 13 & 11 & & \\
\hline \multicolumn{6}{|l|}{ Pathologic type } \\
\hline $\mathrm{ADC}$ & 32 & 15 & 17 & 0.158 & 0.776 \\
\hline SCC & 19 & 10 & 9 & & \\
\hline \multicolumn{6}{|l|}{ pT stage } \\
\hline $\mathrm{T} 1+\mathrm{T} 2$ & 31 & 19 & 12 & 4.763 & 0.029 \\
\hline $\mathrm{T} 3+\mathrm{T} 4$ & 20 & 6 & 14 & & \\
\hline \multicolumn{6}{|l|}{ pN stage } \\
\hline No & 31 & 18 & 13 & 2.588 & 0.093 \\
\hline $\mathrm{N} 1+\mathrm{N} 2$ & 20 & 7 & 13 & & \\
\hline \multicolumn{6}{|l|}{ Differentiation } \\
\hline Well+Moderate & 32 & 18 & 14 & 1.797 & 0.147 \\
\hline Poor & 19 & 7 & 12 & & \\
\hline
\end{tabular}

miRNAs play important roles in tumor progression [28-30]. Recently, miR-497 was found to be down-regulated in a variety of cancers, such as primary peritoneal carcinoma, lung cancer, gastric cancer, malignant pleural mesothelioma and breast cancer [11, 31-34]. Lei Shen reported that miR-497 induces apoptosis of breast cancer cells by targeting Bcl-w. MiR-497 was also found to modulate multidrug resistance of human cancer cell lines by targeting BCL2 [31]. In this study, we found that miR-497 was expressed at lower levels in NSCLC tumor tissues. Low miR-497 levels correlated with advanced pT staging and poor survival. The down-regulation of miR-497 expression led to elevated proliferation activity. Moreover, the overexpression of miR-497 caused a reduced growth rate. These results suggest that miR-497 is a tumor suppressor, as previously reported [32]. In addition, we performed dual luciferase reporter assays and confirmed that miR-497 can directly regulate YAP1 expression by binding to the 3'-UTR of the YAP1 gene. These results suggest that miR497 may inhibit tumor proliferation via inversely regulating YAP1. To verify this hypothesis, we performed a rescue assay. We found that exogenous YAP1 could greatly abrogate the miR497-induced inhibition of proliferation.

Although we indicated the clinical significance of miR-497 in patients with NSCLC, this work was based on a retrospective study. Future work is needed to verify the proliferationinhibiting effect of the miR-497-YAP1 axis in vivo. In summary, this study suggests that miR497 is down-regulated in NSCLC and thus could inhibit NSCLC proliferation via suppressing YAP1 expression. Our data imply that miR-497 may be a potential therapeutic target in NSCLC treatment.

\section{Acknowledgments}

Science Foundation of Tianjin Health Bureau (2011KZ79); National Natural Science Foundation of China (81302487); Wu Jieping Clinical Medical Foundation (320.6750.13129).

\section{Disclosure Statement}

The authors declare that they have no conflict of interest. 


\section{Cellular Physiology Cell Physiol Biochem 2015;37:342-352 \begin{tabular}{ll|l} 
and Biochemistry & $\begin{array}{l}\text { DOI: 10.1159/000430358 } \\
\text { Published onlne: August 24, } 2015\end{array}$ & $\begin{array}{l}\text { O) 2015 S. Karger AG, Basel } \\
\text { www.karger.com/cpb }\end{array}$ \\
\cline { 2 - 3 }
\end{tabular}}

Huang et al.: MiR-497/YAP1 Axis Inhibits NSCLC Growth

\section{References}

1 Siegel R, Naishadham D, Jemal A: Cancer statistics, 2013. CA Cancer J Clin 2013;63:11-30.

-2 Zhang L, Qian J, Qiang Y, Huang H, Wang C, Li D, Xu B: Down-regulation of mir-4500 promoted non-small cell lung cancer growth. Cell Physiol Biochem 2014;34:1166-1174.

3 Li C, Hong W: Research status and funding trends of lung cancer biomarkers. J Thorac Dis 2013;5:698.

4 Wu N, Zhang C, Bai C, Han YP, Li Q: Mir-4782-3p inhibited non-small cell lung cancer growth via usp14. Cell Physiol Biochem 2014;33:457-467.

5 Chen J-F, Mandel EM, Thomson JM, Wu Q, Callis TE, Hammond SM, Conlon FL, Wang D-Z: The role of microrna-1 and microrna-133 in skeletal muscle proliferation and differentiation. Nat Genet 2005;38:228233.

6 Xia Y, Chen Q Zhong Z, Xu C, Wu C, Liu B, Chen Y: Down-regulation of mir-30c promotes the invasion of non-small cell lung cancer by targeting mta1. Cell Physiol Biochem 2013;32:476-485.

-7 Naguibneva I, Ameyar-Zazoua M, Polesskaya A, Ait-Si-Ali S, Groisman R, Souidi M, Cuvellier S, HarelBellan A: The microrna mir-181 targets the homeobox protein hox-a11 during mammalian myoblast differentiation. Nat Cell Biol 2006;8:278-284.

8 Ma L, Teruya-Feldstein J, Weinberg RA: Tumour invasion and metastasis initiated by microrna-10b in breast cancer. Nature 2007;449:682-688.

9 Petrocca F, Visone R, Onelli MR, Shah MH, Nicoloso MS, De Martino I, Iliopoulos D, Pilozzi E, Liu C-G, Negrini M: E2f1-regulated micrornas impair tgf $\beta$-dependent cell-cycle arrest and apoptosis in gastric cancer. Cancer Cell 2008;13:272-286.

10 Iorio MV, Casalini P, Tagliabue E, Ménard S, Croce CM: Microrna profiling as a tool to understand prognosis, therapy response and resistance in breast cancer. Eur J Cancer 2008;44:2753-2759.

11 Li D, Zhao Y, Liu C, Chen X, Qi Y, Jiang Y, Zou C, Zhang X, Liu S, Wang X: Analysis of mir-195 and mir-497 expression, regulation and role in breast cancer. Clin Cancer Res 2011;17:1722-1730.

12 Shen L, Li J, Xu L, Ma J, Li H, Xiao X, Zhao J, Fang L: Mir-497 induces apoptosis of breast cancer cells by targeting bcl-w. Exp Ther Med 2012;3:475-480.

13 Hao Y, Chun A, Cheung K, Rashidi B, Yang X: Tumor suppressor lats1 is a negative regulator of oncogene yap. J Biol Chem 2008;283:5496-5509.

14 Zhao B, Ye X, Yu J, Li L, Li W, Li S, Yu J, Lin JD, Wang C-Y, Chinnaiyan AM: Tead mediates yap-dependent gene induction and growth control. Genes Dev 2008;22:1962-1971.

15 Zhao W-y, Wang Y, An Z-j, Shi C-g, Zhu G-a, Wang B, Lu M-y, Pan C-k, Chen P: Downregulation of mir-497 promotes tumor growth and angiogenesis by targeting hdgf in non-small cell lung cancer. Biochem Biophys Res Commun 2013;435:466-471.

16 Lim LP, Lau NC, Garrett-Engele P, Grimson A, Schelter JM, Castle J, Bartel DP, Linsley PS, Johnson JM: Microarray analysis shows that some micrornas downregulate large numbers of target mrnas. Nature 2005;433:769-773.

17 Yu S-J, Hu J-Y, Kuang X-Y, Luo J-M, Hou Y-F, Di G-H, Wu J, Shen Z-Z, Song H-Y, Shao Z-M: Microrna-200a promotes anoikis resistance and metastasis by targeting yap1 in human breast cancer. Clin Cancer Res 2013;19:1389-1399.

18 Yadav S, Pandey A, Shukla A, Talwelkar SS, Kumar A, Pant AB, Parmar D: Mir-497 and mir-302b regulate ethanol-induced neuronal cell death through bcl2 protein and cyclin d2. J Biol Chem 2011;286:3734737357.

19 Luo Q, Li X, Gao Y, Long Y, Chen L, Huang Y, Fang L: Mirna-497 regulates cell growth and invasion by targeting cyclin e1 in breast cancer. Cancer Cell Int 2013;13:95.

20 Rosenbluh J, Nijhawan D, Cox AG, Li X, Neal JT, Schafer EJ, Zack TI, Wang X, Tsherniak A, Schinzel AC: B-catenin-driven cancers require a yap1 transcriptional complex for survival and tumorigenesis. Cell 2012;151:1457-1473.

21 Yu J, Cheng YY, Tao Q, Cheung KF, Lam CN, Geng H, Tian LW, Wong YP, Tong JH, Ying JM: Methylation of $<$ i> protocadherin $10</ \mathrm{i}>$, a novel tumor suppressor, is associated with poor prognosis in patients with gastric cancer. Gastroenterology 2009;136:640-651.

22 Wojtowicz JM, Kee N: Brdu assay for neurogenesis in rodents. Nat Protoc 2006;1:1399-1405.

23 Kapoor A, Yao W, Ying H, Hua S, Liewen A, Wang Q Zhong Y, Wu C-J, Sadanandam A, Hu B: Yap1 activation enables bypass of oncogenic kras addiction in pancreatic cancer. Cell 2014;158:185-197. 


\section{Cellular Physiology Cell Physiol Biochem 2015;37:342-352

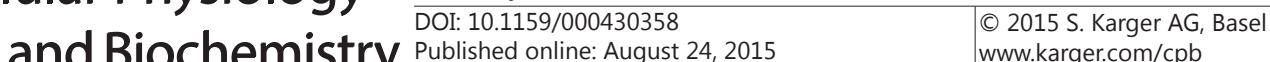 www.karger.com/cpb \\ Huang et al.: MiR-497/YAP1 Axis Inhibits NSCLC Growth}

-24 Camargo FD, Gokhale S, Johnnidis JB, Fu D, Bell GW, Jaenisch R, Brummelkamp TR: Yap1 increases organ size and expands undifferentiated progenitor cells. Curr Biol 2007;17:2054-2060.

-25 Shao DD, Xue W, Krall EB, Bhutkar A, Piccioni F, Wang X, Schinzel AC, Sood S, Rosenbluh J, Kim JW: Kras and yap1 converge to regulate emt and tumor survival. Cell 2014;158:171-184.

-26 Zhao B, Wei X, Li W, Udan RS, Yang Q, Kim J, Xie J, Ikenoue T, Yu J, Li L: Inactivation of yap oncoprotein by the hippo pathway is involved in cell contact inhibition and tissue growth control. Genes Dev 2007;21:2747-2761.

-27 Li M, Lu J, Zhang F, Li H, Zhang B, Wu X, Tan Z, Zhang L, Gao G, Mu J: Yes-associated protein 1 (yap1) promotes human gallbladder tumor growth via activation of the axl/mapk pathway. Cancer letters 2014;355:201-209.

28 Hu X, Zhang F, Liu XR, Wu YT, Ni YM: Efficacy and potential microrna mechanism for computed tomography-guided percutaneous radiofrequency ablation of primary lung cancer and lung metastasis from liver cancer. Cell Physiol Biochem 2014;33:1261-1271.

-29 Wang J, Yang B, Han L, Li X, Tao H, Zhang S, Hu Y: Demethylation of mir-9-3 and mir-193a genes suppresses proliferation and promotes apoptosis in non-small cell lung cancer cell lines. Cell Physiol Biochem 2013;32:1707-1719.

-30 Zhang H, Zhang H, Zhao M, Lv Z, Zhang X, Qin X, Wang H, Wang S, Su J, Lv X, Liu H, Du W, Zhou W, Chen X, Fei K: Mir-138 inhibits tumor growth through repression of ezh2 in non-small cell lung cancer. Cell Physiol Biochem 2013;31:56-65.

-31 Zhu W, Zhu D, Lu S, Wang T, Wang J, Jiang B, Shu Y, Liu P: Mir-497 modulates multidrug resistance of human cancer cell lines by targeting bcl2. Medical Oncology 2012;29:384-391.

-32 Guo J, Miao Y, Xiao B, Huan R, Jiang Z, Meng D, Wang Y: Differential expression of microrna species in human gastric cancer versus non-tumorous tissues. Eur J Gastroenterol Hepatol 2009;24:652-657.

-33 Flavin RJ, Smyth PC, Laios A, O'Toole SA, Barrett C, Finn SP, Russell S, Ring M, Denning KM, Li J: Potentially important microrna cluster on chromosome 17p13. 1 in primary peritoneal carcinoma. Modern Pathology 2008;22:197-205.

34 Balatti V, Maniero S, Ferracin M, Veronese A, Negrini M, Ferrocci G, Martini F, Tognon MG: Micrornas dysregulation in human malignant pleural mesothelioma. J Thorac Oncol 2011;6:844-851. 